JCMS

3,2

98

Received 28 May 2019

Revised 9 August 2019 23 August 2019 Accepted 27 August 2019

\section{Cryptocurrencies: applications and investment opportunities}

\author{
A. Can Inci \\ Department of Finance, Bryant University, Smithfield, Rhode Island, USA, and \\ Rachel Lagasse \\ Mathworks Inc, Natick, Massachusetts, USA
}

\begin{abstract}
Purpose - This study investigates the role of cryptocurrencies in enhancing the performance of portfolios constructed from traditional asset classes. Using a long sample period covering not only the large value increases but also the dramatic declines during the beginning of 2018, the purpose of this paper is to provide a more complete analysis of the dynamic nature of cryptocurrencies as individual investment opportunities, and as components of optimal portfolios.

Design/methodology/approach - The mean-variance optimization technique of Merton (1990) is applied to develop the risk and return characteristics of the efficient portfolios, along with the optimal weights of the asset class components in the portfolios.

Findings - The authors provide evidence that as a single investment, the best cryptocurrency is Ripple, followed by Bitcoin and Litecoin. Furthermore, cryptocurrencies have a useful role in the optimal portfolio construction and in investments, in addition to their original purposes for which they were created. Bitcoin is the best cryptocurrency enhancing the characteristics of the optimal portfolio. Ripple and Litecoin follow in terms of their usefulness in an optimal portfolio as single cryptocurrencies. Including all these cryptocurrencies in a portfolio generates the best (most optimal) results. Contributions of the cryptocurrencies to the optimal portfolio evolve over time. Therefore, the results and conclusions of this study have no guarantee for continuation in an exact manner in the future. However, the increasing popularity and the unique characteristics of cryptocurrencies will assist their future presence in investment portfolios.

Originality/value - This is one of the first studies that examine the role of popular cryptocurrencies in enhancing a portfolio composed of traditional asset classes. The sample period is the largest that has been used in this strand of the literature, and allows to compare optimal portfolios in early/recent subsamples, and during the pre-/post-cryptocurrency crisis periods.
\end{abstract}

Keywords Bitcoin, Ripple, Portfolio optimization, Cryptocurrencies, Litecoin, Markowitz

Paper type Research paper

\section{Introduction}

Cryptocurrencies are a basic topic of understanding to some, and a complete mystery to many. Throughout the past few years, these digital currencies have become the next big thing for select investors, but a topic of complete confusion and question for others. What are cryptocurrencies, and why have they become such a large topic for discussion as of recently? In the last quarter of 2017, this question was asked widely, as Bitcoin surged in value more than 2,500 percent from mid-December 2016 to mid-December 2017. The interest on cryptocurrencies increased even more after the great cryptocurrency crash in the beginning of 2018. In the course of one day, Bitcoin lost about 15 percent of its value, dropping from $\$ 13,500$ to $\$ 11,500$ in a matter of hours (Burgess, 2018). Although Bitcoin is the most well-known digital currency, it is just one of the more than 1,500 cryptocurrencies.

\section{JEL Classification — G01, G11, O16, O33}

(C) A. Can Inci and Rachel Lagasse. Published in Journal of Capital Markets Studies. Published by Emerald Publishing Limited. This article is published under the Creative Commons Attribution (CC BY 4.0) licence. Anyone may reproduce, distribute, translate and create derivative works of this article (for both commercial and non-commercial purposes), subject to full attribution to the original publication and authors. The full terms of this licence may be seen at http://creativecommons.org/ licences/by/4.0/legalcode
Journal of Capital Markets Studies Vol. 3 No. 2, 2019 pp. $98-112$

Emerald Publishing Limited 2514-4774

DOI 10.1108/JCMS-05-2019-0032 
Other popular cryptocurrencies with the largest market capitalizations are Ethereum, Ripple and Litecoin. Cryptocurrencies operate differently and are distinguished from one another mainly due to their values, transaction speeds, usages and volatility characteristics.

Although cryptocurrencies were not originally created as investment assets, many investors use them as such. Due to the fact that cryptocurrencies are still relatively new in the time frame of the financial securities world, there has not been extensive research done on the effects of including them in a portfolio. This study aims to find diversification benefits of cryptocurrencies, and how they contribute to an optimal investment portfolio.

Our sample period covers most of the 2010s. We examine the most popular cryptocurrencies: Bitcoin, Ripple and Litecoin. We document that as an independent single investment, Ripple has the highest return, followed by Litecoin and Bitcoin. However, volatility of Ripple is also the highest, followed by those of Litecoin and Bitcoin. Combining risk and return together, the coefficient of variations reveal that the best cryptocurrency is Ripple, followed by Bitcoin, and then by Litecoin. All these cryptocurrencies have been better for investment purposes than the traditional asset classes in the 2010s.

We also examine the role of cryptocurrencies in optimal portfolios. We document that adding a cryptocurrency has always helped the optimal portfolio to achieve a better return and risk combination. Bitcoin has been the most useful cryptocurrency in this regard, followed by Ripple and Litecoin. We report that the contribution of the cryptocurrencies to an optimal portfolio is dynamic and, therefore, evolves over time. The cryptocurrency crash clearly has led to reduction in the attractiveness of cryptocurrencies as investment alternatives but the dynamic characteristics of cryptocurrencies warrant their future demand in investment portfolios. We conclude in our study that popular cryptocurrencies have had a useful role in portfolio construction and in investments, in addition to their original purposes for which they were created.

We would also like to point out that even though the investment portfolio benefits of cryptocurrencies are clear in the 2000s, the future is always unpredictable and the past results and conclusions need not necessarily extend into the future. From a qualitative perspective, the popularity of and familiarity with cryptocurrencies increase continually. The variety of these investment tools is also expanding. The blockchain technology with which most cryptocurrencies have strong links is getting more and more into the mainstream of institutional and corporate operations. With all these continual developments, it is a safe conjecture that cryptocurrencies will have an important role in investment portfolios. However, the return and covariance characteristics observed and reported in our paper are historical; with no guarantee for continuation in an exact manner into the future.

The rest of the paper is organized as follows. Section 2 presents the literature review with an overview of cryptocurrencies, along with their popularity, problems and their roles in investment portfolios. Section 3 is about research methodology. Section 4 presents the data. Section 5 covers the results and the discussion. Section 6 concludes the paper.

\section{Literature review}

\subsection{Cryptocurrencies overview}

A cryptocurrency can be defined as "cash for the internet." It is a piece of digital information that one can hold onto, and it has value as long as no one else has access to the information and keys that provide the value. The entity who holds the information at that point of time is also the holder of the value of that cryptocurrency.

The creation of cryptocurrencies is based on the need for internet cash combined with the desire for anonymity in internet transactions. As explained in Geiregat (2018), scientists and activists concerned with privacy and personal liberty started the experimentation and eventually the invention of the cryptocurrency: an unregulated, decentralized, completely 
JCMS

3,2

anonymous system of transactions directly from user to user (peer-to-peer) without a bank account or credit card.

Cryptocurrencies have three core characteristics: decentralized, unregulated and anonymous. In his manifesto, Nakamoto (2008) highlighted the problems of lack of privacy and safety in transactions, and proposed a well-thought out solution: the blockchain. Decentralization of cryptocurrencies originates from the nature of the blockchain technology. Being a distributed ledger, operated within peer-to-peer networks, the data and information contained on the blockchain platform are available to and reside in thousands of computers all over the world, so that any user, miner or bystander has access to the data. This makes the system highly unlikely to be hacked compared to a centralized organization or system, such as a bank, where there is one central repository of information susceptible to breaches in security. Biais et al. (2019) model the blockchain protocol as a stochastic game and analyze the equilibrium strategies of rational, strategic miners by mining the longest chain as a perfect Markov equilibrium in line with Nakamoto (2008). The blockchain protocol, however, is also a coordination game, with multiple equilibria, hence, the decentralization characteristic.

Blockchain technology and its solicitation through cryptocurrencies provide decentralized consensus and potentially enlarge the contracting space through smart contracts. At the same time, generating decentralized consensus entails distributing information that necessarily alters the informational environment. Cong and He (2019) analyze how decentralization relates to consensus quality and how the quintessential features of blockchain remold the landscape of competition. Smart contracts can mitigate informational asymmetry and improve welfare and consumer surplus through enhanced entry and competition. In general, blockchains sustain market equilibria with a wider range of economic outcomes.

The second key characteristic of the cryptocurrency is the lack of regulation. No government or organization has any control or say over any cryptocurrency, which makes them attractive for many reasons. Because there is no government monitoring, transactions are not subject to sales tax. Moreover, since no one is regulating transactions on a federal or other level, no intermediary such as lawyers, banks or payment providers is needed in the user-to-user system. These aspects of cryptocurrencies open them to potential issues with fraud and to interference by governments in the future.

The third key characteristic of cryptocurrencies is that they are anonymous and untraceable. When a transaction occurs on the blockchain network, each user involved in that transaction has a specific personal key, similar to a user name. Once that transaction is completed and verified, those personal keys are also completed and can never be used again. Every time a user engages in a transaction, a unique and untraceable personal key is generated. Moreover, there is no need for the user to engage a bank account or a credit card for the transaction, keeping the anonymity. This key anonymity characteristic also opens the door to more potential problems for cryptocurrencies.

\subsection{Issues and problems of cryptocurrencies}

Cryptocurrencies have been among the largest unregulated markets in the world. Foley et al. (2019) document that roughly one fourth of Bitcoin users may have been involved in illegal activity. Their study estimates that about $\$ 76 \mathrm{bn}$ of illegal activity each year may involve Bitcoin. They also document that the illegal share of Bitcoin activity has declined with mainstream interest in Bitcoin and with the emergence of more opaque cryptocurrencies. Practical problems related to investing in cryptocurrencies include illiquidity, theft, fraud, ransom attacks/hacking and potential constrictive government regulation. Since cryptocurrencies are unregulated, decentralized, untraceable and anonymous, there are no protections, liability clauses or insurers. 
Although the lack of regulation is an important problem for the lack of protection from theft and ransom attacks, increased levels of regulation could pose an even bigger problem for these digital coins. Government regulation could disrupt the true nature of cryptocurrencies that makes them attractive to users, could lead to drastic declines in their value, and could cause significant illiquidity, making them unattractive to investors. Overall, the potential for regulation is a major threat to cryptocurrencies for the near future.

\subsection{Popular cryptocurrencies}

The top four cryptocurrencies in terms of market capitalization are Bitcoin, Ethereum, Ripple and Litecoin. Bitcoin holds the largest market capitalization allocating half of the cryptocurrency market. Ethereum and Ripple also hold significant spots covering 10 percent of the market capitalization each. Litecoin is on the rise with a smaller holding of the market capitalization of roughly 3 percent. These four cryptocurrencies constitute three fourths of the entire market. As reported in Radovanov et al. (2018), although correlations vary between these cryptocurrencies, they are fundamentally different from each other because of their unit values, market capitalizations and user applications.

Bitcoin has been the most talked about, popular and sophisticated cryptocurrency (Velde, 2013). Launched in 2009, the value has grown tenfold from 2013 to 2018. Figure 1 depicts the Bitcoin price vs the Dow Jones Industrial Average in 2010s. We can see the exponential increase in the Bitcoin value and the dramatic decline during the cryptocurrency crash of January 2018. In the Nakamoto (2008) manifesto outlining the idea of Bitcoin and blockchain, a low-cost secure payment system has been proposed that does not involve a central authority or trusted third party. As explained further in Velde (2013), Bitcoin is not a claim to a physical object or to a currency; rather, it aims to be a currency itself to replace the usual physical object of a currency with a computer file. Bitcoin and other cryptocurrencies use the blockchain network but they differ in from each other terms in terms of the difficulty in mining the specific currency. Bitcoin has a specific hash rate of verifying transactions, a certain number of miners and a goal of six blocks to be created per hour (for an average transaction speed of ten minutes), making it organically fit the blockchain network. Overall, Bitcoin has been primarily utilized as a means to transfer funds within the blockchain environment, but also as a speculative investment opportunity given that the cryptocurrency derives its value from exchange.

Second in the market capitalization in the cryptocurrency market is Ethereum, created in 2014. Ether, the digital coin of Ethereum, is rival to Bitcoin due to the promise of the technology it is built in. Instead of being used as a digital currency, or an alternative to fiat



Figure 1.

Dow Jones industrial average index and the Bitcoin price in US dollars 
JCMS

3,2

money, Ethereum has been built for smart contracts and for decentralized applications. Smart contracts are agreements in the blockchain network that function like software programs that can bind obligations based on predetermined conditions. Ethereum's built-in programming language allows anyone to build augmented applications. The Ethereum network has the potential to host numerous functions such as social networks, public utility applications, crowd-sourced prediction markets and investment companies. Ethereum's ability to create new social structures within a completely virtual network sets it apart from Bitcoin and other cryptocurrencies.

The third cryptocurrency in market capitalization is Ripple, created in 2013 with the primary purpose of helping banks transfer cash faster and cheaper, especially internationally. Traditional methods of cross-border payments are slow, of low value, of high volume and have high transaction costs. Ripple is primarily meant to be used as a currency translation tool, and to be a catalyst, setting it apart from other cryptocurrencies.

The fourth popular cryptocurrency is Litecoin, created in 2011, mainly as an alternative and as an improvement to Bitcoin with faster settlement times for transactions and with lower fees. Litecoin is used for the same purpose like Bitcoin as a method of payment and as an exchange of funds but with better performance for micropayments.

\subsection{Cryptocurrencies in investment portfolios}

There are studies that look into the effects of using Bitcoin to diversify an investment portfolio, such as Wu and Pandey (2014), Klabbers (2017) and Andrianto and Diputra (2017). These studies primarily focus only on Bitcoin. Using dated and limited sample periods from 2010 to 2013 in Wu and Pandey (2014), from 2013 to 2016 in Andrianto and Diputra (2017) and from 2010 to 2016 in Klabbers (2017), the usefulness of Bitcoin as an investment asset in enhancing the efficiency of an investment portfolio were documented.

Lack of sufficient information and the novelty of cryptocurrencies lead to discomfort in the opinions of different investors and financial experts in using Bitcoin and other cryptocurrencies in an investment portfolio (Burgess, 2018). Burgess (2018) documents one expert recommending allocations of no more than 2 percent of a portfolio to Bitcoin due to high-volatility, and not recommending for anyone nearing retirement. Investors generally consider cryptocurrencies as high risk investments and tend to keep some in the portfolio only as an insurance policy: in order to settle ransomware attacks with these cryptocurrencies.

The academic studies mentioned above demonstrate the usefulness of Bitcoin in portfolio efficiency; however, the attitude of investors in general has been lukewarm. To reconcile these contrasting views, we use a more recent and longer sample period ranging from August 2013 through January 2019. Furthermore, we use multiple cryptocurrencies and broad traditional asset classes. We document the dynamic nature of the contribution of cryptocurrencies in a portfolio. We demonstrate that all cryptocurrencies contribute to an efficient portfolio, regardless of the type of cryptocurrency. Our results emphasize the evolving dynamics of return-risk characteristics, while Bitcoin and Ripple held larger positions in optimal portfolios, Litecoin has taken over in recent years, especially after the cryptocurrency crash of January 2018.

Briere et al. (2015) analyze investments in Bitcoin by US investors within a diversified portfolio of both traditional and alternative assets. Over the period of the analysis, Bitcoin had an exceptionally high average return and volatility, and correlation with other assets was low, proving that it had high diversification benefits. However, the conclusion made clear that these results should be looked at cautiously, as the data in this study reflected the early-stage behavior of Bitcoin and might not appropriately represent the performance of the portfolio in the long or even medium run. Overall, there have been some studies conducted on the effect of Bitcoin in enhancing portfolio diversification. But these studies focus only on Bitcoin and not on a combination of other cryptocurrencies; are mostly 
outdated; and use short sample periods with a short-term outlook, not taking into account Cryptocurrencies the long-term volatility effects nor the possibility of increased regulation.

Along the lines of portfolio diversification with more than one cryptocurrency, Brauneis and Mestel (2019) focus on a portfolio made up of only cryptocurrencies and they present evidence of substantial risk reduction. In an attempt to address and quantify the portfolio effects in the cryptocurrency investment universe, they rely on the traditional mean-variance framework as proposed by Markowitz (1952). There is a considerable body of literature that proposes alternatives to the mean-variance optimization when returns are not normal. However, seminal papers such as Levy and Markowitz (1979) and Kroll et al. (1984) demonstrate the equivalence of the mean-variance approach to expected utility maximization under non-normality. In this study, we follow the same principles laid out in Brauneis and Mestel (2019) and provide preliminary evidence on the portfolio effects of multiple cryptocurrency investments. However, our portfolio structure, unlike theirs, is more flexible and general: we examine the most important cryptocurrencies, but we include them in a portfolio of traditional asset classes: equity, bond, real estate and volatility, mainly considering a US based investor (equity is represented by the US stock market), who is somewhat sophisticated (with a derivatives proxy based on volatility index). We examine this overall portfolio through the mean-variance framework.

Anyfantaki et al. (2018) examine the role of cryptocurrencies in a traditional portfolio mainly from a theoretical perspective. Their empirical analysis covers a two-year period from mid-2015 to mid-2017, which is significantly shorter than ours, but provides similar evidence of diversification benefits of cryptocurrencies both in- and out-of-sample. Klein et al. (2018) compare Bitcoin to gold and examine the role of each financial security in a traditional portfolio. They find that Bitcoin exhibits very different properties with respect to gold in that, while gold is useful during down times, Bitcoin is hardly helpful during those same periods.

Limited number of previous studies finds that in the short-term investment horizon, cryptocurrencies may offer diversification benefits. For example, Corbet et al. (2018) look into investing in Bitcoin, Ripple and Litecoin, along with other traditional and alternative financial assets, and document Bitcoin's impact on the other cryptocurrencies. Smaller currencies like Ripple and Litecoin, although have no effect on the value of Bitcoin, are highly interconnected amongst themselves and influence one another's values. Results from the Corbet et al. (2018) study show that cryptocurrencies are "isolated from other markets," and general market conditions are not highly influential on cryptocurrencies. Therefore, cryptocurrencies do present diversification benefits as they are disconnected from the general financial markets. However, the cryptocurrency market contains its own idiosyncratic risks that are not easy to hedge against. The Corbet et al. (2018) study mainly explores the time series relationships and correlations amongst cryptocurrencies and between cryptocurrency - regular asset pairs. In our study, we focus on the optimal portfolio characteristics with cryptocurrencies as part of portfolios.

Elendner et al. (2018) provide a qualitative description of cryptocurrency portfolios. They examine numerous portfolios of cryptocurrencies, as well as the cryptocurrency index, CRIX and evaluate the performance of these portfolios. They also consider equally weighted and value-weighted broad portfolios made up of cryptocurrencies and traditional assets. They provide evidence of diversification benefits of cryptocurrencies because of their limited co-movements with traditional financial securities. However, the optimal portfolio construction is not part of the Elendner et al. (2018) study. Focusing on ten major cryptocurrencies, Liu (2019) examines the role of diversification amongst cryptocurrencies. Evaluation of the out-of-sample performance of this cryptocurrency-only investment spectrum shows that portfolio diversification across different cryptocurrencies is beneficial, implying that each cryptocurrency has unique characteristics. Borri (2019) examines contagion and flight-to-quality characteristics of cryptocurrencies amongst themselves 
JCMS

3,2

104

focusing on the tail-risk events and provides evidence that cryptocurrency portfolios offer better risk-adjusted and conditional returns than individual cryptocurrencies. On the other hand, Bouri et al. (2019) find that common characteristics between cryptocurrencies increase during uncertain periods. In a more rigorous approach by Härdle and Trimborn (2015), the relationship between cryptocurrencies and the cryptocurrency index - CRIX is examined at a theoretical level.

\section{Research methodology}

This paper aims to answer should, and if yes how, cryptocurrencies can be used to diversify an investment portfolio. Additionally, we ask how cryptocurrencies correlate with each other and with other asset classes, and how cryptocurrencies rank as individual investments, taking both risk and return properties into account.

In the portfolio optimization framework, we follow the mean-variance optimization technique outlined in Kroll et al. (1984) and Merton (1990). The Kroll et al. (1984) study compares the mean-variance optimization technique with direct utility maximization with infinite number of alternate probability distributions for the expected utility maximizer. They conclude under standard portfolio constraint sets faced by a wide variety of professional and individual investors that the mean-variance portfolio estimation is a good procedure to determine the optimal portfolio for investment purposes.

We follow the portfolio optimization methods based on the Merton proposition involving envelope portfolios (Merton, 1990). This method finds the optimal weights of each asset class on the tangent portfolio of the efficient frontier. Using the average daily returns and the variance-covariance characteristics of the financial assets through linear algebra and matrix operations, the optimal weights are assigned to each asset in the portfolio to establish the most efficient portfolio possible.

The main conjecture of this study is that cryptocurrencies, when considered as financial securities, would provide enhancements to portfolio with a higher return and/or lower risk because of their unique and different characteristics compared to traditional financial assets. We also conjecture that amongst themselves, cryptocurrencies have been quite different; therefore, all three that we examine in this paper would have contributions to the optimal portfolio.

\section{Data}

Daily price data for seven different asset classes are used in the analyses. We consider cryptocurrencies as financial securities for investment portfolios. For the three cryptocurrencies, the data are obtained from Coin Metrics. The price data for the other four asset classes are collected from FactSet. We focus on Bitcoin, Ripple and Litecoin because they have been in existence the longest. Our sample period with daily frequency is from August 5, 2013 through January 17, 2019, covering the majority of 2010s.

The seven asset classes chosen for the optimization are three cryptocurrencies: Bitcoin (BTC), Ripple (XRP), Litecoin (LTC); equity represented by the Dow Jones Industrial Average; real estate represented by the Vanguard Real Estate Index; fixed income securities represented by the Vanguard Total Bond Market Index; and a derivatives proxy utilized by sophisticated investors represented by the CBOE Volatility Index. It should be noted that Ethereum was not used in the study since, although it is a popular cryptocurrency, it came into existence quite recently in 2014 .

Once the price data on all the seven asset classes were collected from the two different sources, they were compiled into one cohesive data frame and incomplete information due to differences in the dates of the data for the cryptocurrencies was eliminated. Daily returns are calculated using:

$$
r(t)=\ln (P(t))-\ln (P(t-1))
$$


where $\ln (P(t)$ and $\ln (P(t-1)$ are the natural logarithms of the closing prices on day $t$ and Cryptocurrencies day $t-1$.

\subsection{The portfolio optimization technique}

The particular portfolio optimization analysis involves linear algebra and matrix operations. The weights of the optimal portfolio are obtained in accordance with the Merton (1990) proposition, which states that all envelope portfolios in the financial securities universe solve the following equation:

$$
x=\frac{S^{-1}\{E(R)-c\}}{\operatorname{Sum}\left(S^{-1}\{E(R)-c\}\right)},
$$

where $x$ is the vector of optimal weights of the components of the portfolio (with these weights adding up to 1 ), $S$ is variance-covariance matrix, $E(R)$ is the expected return vector composed of anticipated daily returns of the components of the portfolio, and $c$ is an arbitrary constant. Any constant $c$ leads to an envelope frontier portfolio. The denominator of the right hand side of Equation (2) normalizes the numerator and makes sure that the optimal weights add up to 1 .

The Merton (1990) proposition gives a specific method of finding the tangency portfolio, given $c$. In that specific optimization, the constant $c$ is economically interpreted as the risk-free rate of return, $r_{f}$. The variance-covariance matrix, $S$, represents the risk characteristics of the investment portfolio. It is defined as a diagonal and symmetric matrix, where the diagonal elements are variances of the individual financial securities, and the off-diagonal elements are the covariances between financial security pairs. Hence, the formal name of the $S$ is the variance-covariance matrix.

The steps in this portfolio optimization procedure are:

- creation of the return vector where each element is the average of the daily returns of the financial securities in the portfolio;

- creation of the variance-covariance matrix, $S$, using the entire data set of the returns of all the investments in the portfolio; and

- determination of the optimal weights vector, $x$, following the Merton (1990) proposition summarized in Equation (2).

Once the initial optimization was completed using all of the data collected, multiple other robustness analyses were conducted with different portfolios through the inclusion and exclusion of asset classes. Further tests were also conducted by covering different sample periods. These tests and their results are discussed in the following section.

\section{Results and discussion}

The portfolio with the traditional asset classes in equity, fixed income, real estate, along with the volatility index representing derivatives market for sophisticated investors is further enhanced with the cryptocurrencies, Bitcoin, Ripple and Litecoin. Table I presents the summary statistics of the return characteristics of these financial securities for the sample period covering August 2013-January 2019.

We see that the cryptocurrencies generate the highest daily average raw returns during the period of investigation. Ripple has the highest return, followed by Litecoin and Bitcoin. Volatility index average return is also high (in fact, higher than the Bitcoin return). Thus, we can say that during most of the 2010s, traditional asset classes have generated lower returns than the popular cryptocurrencies. Examination of the volatilities of these investments also 
JCMS

3,2

106

shows, however, that the cryptocurrencies have higher volatilities compared to those of other investment alternatives.

In order to take into account the volatility of the assets as well as the returns, we focus on $\mathrm{CV}$ - the coefficient of variation, which is defined as the ratio of standard deviation of daily returns to the average of the returns. The lower the $\mathrm{CV}$, the better the investment, taking both risk and return into account. As we can see from Table I, the popular cryptocurrencies have the lowest CVs compared to the other asset classes during the 2010s, indicating their superiority as investment alternatives. The best cryptocurrency in this regards is Ripple, followed by Bitcoin and then by Litecoin. The remaining asset classes in terms of attractiveness are US equities, volatility index, bonds and real estate.

Before moving on with the construction of the optimal portfolio, it is useful to get an overall view of the correlations between the financial securities during the 2010s. Table II presents the correlation matrix, where the Pearson correlation coefficient for every asset pair is listed. As one can observe from the correlation matrix, there are indeed benefits to forming portfolios during the investigation period. The low correlations within the asset pairs, the negative correlation values and the evidence of no relationship between equity returns and cryptocurrency returns are all indications of the advantages and the rational necessity of forming portfolios of these securities during the majority of the 2010s period. Volatility-based investment opportunities such as VIX based financial products naturally have high negative correlations with equity markets. As for cryptocurrency correlations between themselves, the highest positive correlations have been between Bitcoin and Litecoin.

The results from the correlation table enable the construction of portfolios enhancing return and reducing risk, potentially at the same time. According to this conjecture, the CV of such portfolios should be lower than any of the individual asset classes examined in this

\begin{tabular}{lrrrrrrr}
\hline & BTC & \multicolumn{1}{c}{ XRP } & \multicolumn{1}{c}{ LTC } & \multicolumn{1}{c}{ DJIA } & \multicolumn{1}{c}{ VIX } & Real Estate & Bonds \\
\hline Mean & 0.0030 & 0.0062 & 0.0037 & 0.0004 & 0.0037 & 0.0003 & 0.0001 \\
SD & 0.0457 & 0.0934 & 0.0760 & 0.0083 & 0.0864 & 0.0093 & 0.0021 \\
CV & 15.2327 & 15.1847 & 20.5730 & 23.2187 & 23.6154 & 29.3116 & 24.0852 \\
Skewness & 0.8838 & 6.1717 & 5.0947 & -0.3823 & 2.7721 & -0.3996 & -0.0928 \\
Kurtosis & 12.2464 & 97.3171 & 70.0579 & 3.8674 & 26.6584 & 1.7195 & 0.4888
\end{tabular}

Notes: BTC is Bitcoin, XRP is Ripple, LTC is Litecoin, DJIA is Dow Jones Industrial Average Index, Real Estate is the Vanguard Real Estate Index, and Bonds is the Vanguard Total Bond Market Index. CV is the coefficient of variation. Daily raw price values and raw return values are used for the statistical calculations in the table. The sample period is from August 4, 2013 through January 17, 2019

Table I.
Summary statistics

\begin{tabular}{lrrrrrr}
\hline & BTC & XRP & LTC & DJIA & VIX & Real Estate \\
\hline XRP & 0.31 & & & & & \\
LTC & 0.67 & 0.34 & & & & \\
DJIA & 0.05 & 0.02 & 0.03 & & & \\
VIX & -0.05 & -0.01 & -0.03 & -0.77 & & \\
Real Estate & 0.03 & 0.02 & 0.02 & 0.55 & -0.46 & \\
Bonds & -0.01 & 0.04 & -0.01 & -0.31 & 0.25 & 0.13
\end{tabular}

Notes: BTC is Bitcoin, XRP is Ripple, LTC is Litecoin, DJIA is Dow Jones Industrial Average Index, Real Estate is the Vanguard Real Estate Index and Bonds is the Vanguard Total Bond Market Index. The sample

Table II.

Correlation matrix period is from August 4, 2013 through January 17, 2019. Daily returns are used for the Pearson correlation values in the table 
paper during the same sample period. To explore this conjecture, we follow the Merton (1990) approach and construct the optimal portfolio using these seven (three popular cryptocurrencies, three traditional investments and one derivatives proxy) investment alternatives. The optimal portfolio would be an efficient portfolio with as much profit and as little risk as possible. By taking into account the risk free rate during the sample period of the portfolio creation, the optimal portfolio would also be the tangent portfolio residing on a straight line starting from the risk free rate and is tangent to the efficient frontier according to portfolio theory. Following the steps of the Merton's proposition for the mean-variance optimization, the results are provided in Table III.

In Panel A of Table III, we see the critical pieces of information for the optimal portfolio. The daily return of the optimal portfolio is 5.1 basis points. The volatility is 32.37 basis points, which is lower than all the individual investment alternatives except for fixed income. Most importantly, the coefficient of variation is 6.35 , and this is much smaller and therefore superior to the individual assets (the lowest CV of the individual investments is 15.2 for Ripple). Panel B provides the optimal weights of the portfolio as percentages. The optimal portfolio for the 2013-2019 sample period of the study for the 2010s primarily includes stocks and bonds. Bitcoin and Ripple do have a part in the optimal portfolio with 2.6 and 1.1 percent weights, respectively. Litecoin has a short position with a small negative weight. Given that Bitcoin and Ripple enhance the optimal portfolio in the 2010s period with their positive weights, and Litecoin with a nonzero negative weight, we can conclude that the popular cryptocurrencies have had a role in portfolio construction and in investments, in addition to their original purposes for which they were created.

Finally, Panel $\mathrm{C}$ of Table III provides the variance-covariance matrix, which summarizes the risk features of the components of the optimal portfolio. The diagonal elements of the matrix are the variances of the individual components, while the off-diagonal elements the covariances between investment pairs.

We also conducted some bootstrap analysis following the recommendation of an anonymous referee to determine the weights in an optimal portfolio for a fixed time horizon of 500 business days. We selected individual days' returns randomly with replacement for the original sample for each portfolio component. By selecting the daily returns randomly,

Panel A. Optimal portfolio

$\begin{array}{ccc}\text { Return (bp) } & \text { Std (bp) } & \text { CV } \\ 5.10 & 32.37 & 6.345590\end{array}$

Panel B. Optimal portfolio weights (\%)

$\begin{array}{ccccccr}\text { BTC } & \text { XRP } & \text { LTC } & \text { DJIA } & \text { VIX } & \text { Real estate } & \text { Bonds } \\ 2.5631 & 1.0752 & -0.1375 & 47.7659 & 4.4248 & 0.9423 & 43.3662\end{array}$

Panel C. Optimal portfolio variance-covariance matrix

\begin{tabular}{lrrrrrrr} 
& BTC & XRP & LTC & DJIA & VIX & Real estate & \multicolumn{1}{c}{ Bonds } \\
BTC & 0.002086 & 0.001342 & 0.002323 & 0.000019 & -0.000215 & 0.000015 & -0.000001 \\
XRP & 0.001342 & 0.008733 & 0.002419 & 0.000016 & -0.000101 & 0.000016 & 0.000007 \\
LTC & 0.002323 & 0.002419 & 0.005782 & 0.000021 & -0.000187 & 0.000015 & -0.000001 \\
DJIA & 0.000019 & 0.000016 & 0.000021 & 0.000068 & -0.000548 & 0.000042 & -0.000005 \\
VIX & -0.000215 & -0.000101 & -0.000187 & -0.000548 & 0.007462 & -0.000368 & 0.000045 \\
Real estate & 0.000015 & 0.000016 & 0.000015 & 0.000042 & -0.000368 & 0.000087 & 0.000003 \\
Bonds & -0.000001 & 0.000007 & -0.000001 & -0.000005 & 0.000045 & 0.000003 & 0.000004
\end{tabular}

Notes: Optimal portfolio characteristics with the inclusion of cryptocurrency assets are provided. In Panel A, the return and standard deviation of the optimal portfolio are listed as basis points along with the coefficient of variation. Panel B optimal weights of the seven asset classes are given as percentages. Finally, Panel C is optimal portfolio risk matrix, the variance covariance matrix. The sample period is from August 4, 2013 through January 17, 2019 
JCMS

3,2

108

the cross-sectional covariance structure would be preserved, but we get a better picture of a possible return distribution. Our bootstrapping process has indeed emphasized the importance of the cryptocurrencies: the optimal weights are 5.5 percent for Bitcoin, 3.3 percent for Ripple and 3.1 percent for Litecoin, making up about 12 percent of the portfolio during our sample period according to the bootstrapping procedure.

The optimal portfolio in Table III contained all three popular cryptocurrencies investigated in this study. While in the Table I discussion we have concluded that as a single investment, Ripple is the best cryptocurrency with its smallest $\mathrm{CV}$, followed by Bitcoin and Litecoin, we now investigate how each cryptocurrency individually and separately contributes to the optimal portfolio. In Table IV, we create optimal portfolios with a single cryptocurrency as the fifth portfolio component in addition to the equity, fixed income, real estate and volatility index components. First, the optimal portfolio without any cryptocurrency is presented in Panel A. In addition to the weights of the portfolio components, the return and the standard deviation are provided. Summarizing all, the CV of this portfolio is 7.34 .

Panel B depicts the results of the portfolio augmented with Bitcoin. The weight of Bitcoin in this optimal portfolio is 3.02 percent and this positive weight helps the optimal portfolio achieve a lower CV of 6.60. Panels $\mathrm{C}$ and $\mathrm{D}$ are for portfolios augmented with Ripple (1.5 percent weight) and Litecoin (1.3 percent weight). These portfolios have CVs of 6.68 and 6.93 , respectively. Overall, we can conclude that adding a cryptocurrency has consistently helped the optimal portfolio to achieve a better return and risk combination summarized

$\begin{array}{lccc}\text { Panel A. Optimal portfolio no cryptocurrency } & & \\ \text { Return (bp) } & \text { Std (bp) } & \text { CV } & \\ 3.76 & 27.60 & 7.335607 & \\ \text { Optimal Portfolio Weights (\%) } & & \\ \text { DJIA } & \text { VIX } & \text { Real Estate } & \text { Bonds } \\ \text { 48.6562 } & 4.3891 & 0.9063 & 46.0485\end{array}$

Panel B. Optimal portfolio with BTC

$\begin{array}{llc}\text { Return (bp) } & \text { Std (bp) } & \text { CV } \\ 4.57 & 30.18 & 6.597790\end{array}$

Optimal portfolio weights ( $\%)$

BTC DJIA VIX

$\begin{array}{lll}3.0229 & 47.0488 & 4.3298\end{array}$

46.0485

Panel C. Optimal portfolio with XRP

$\begin{array}{lll}\text { Return (bp) Std (bp) CV } & \end{array}$

$4.72 \quad 31.52 \quad 6.684067$

Optimal Portfolio Weights (\%)

XRP DJIA VIX

$1.4734 \quad 49.1966 \quad 4.5025$

VIX Real Estate Bonds

$1.1189 \quad 43.7086$

Panel D. Optimal portfolio with LTC

$\begin{array}{lll}\text { Return (bp) } & \text { Std (bp) }\end{array}$

$4.21 \quad 29.16 \quad 6.926162$

Optimal Portfolio Weights (\%)

LTC DJIA

$1.3301 \quad 47.8990$

VIX

Real Estate

Bonds

$0.8693 \quad 45.5430$

Notes: Optimal portfolio characteristics with different cryptocurrencies are provided in the table. Panel A does not have any cryptocurrencies. Panel B is the portfolio with Bitcoin as the fifth asset class. Panel $\mathrm{C}$ is the portfolio with Ripple. Panel D is the portfolio with Litecoin. The return and standard deviation of the optimal portfolio are listed as basis points along with the coefficient of variation. Optimal portfolio weights are given as percentages. The sample period is from August 4, 2013 through January 17, 2019 
with a lower CV; Bitcoin has been the best cryptocurrency enhancing the characteristics of the portfolio because the optimal portfolio with Bitcoin has the lowest CV during the sample period; Litecoin has been the least useful cryptocurrency: the CV of the optimal portfolio is lower than the $\mathrm{CV}$ of the original no cryptocurrency portfolio, but higher than the CVs of the portfolios with the other cryptocurrencies.

Are the portfolio contributions of cryptocurrencies static or dynamic over time? To investigate this issue, we split the entire sample into two subsamples: the early subsample from August 4, 2013 through April 26, 2016 and the recent subsample from April 27, 2016 through January 17, 2019. We construct the optimal portfolio using the cryptocurrencies and the traditional assets for each subsample separately. The results reported in Table V provide some interesting observations. The early subsample results in Panel A depict the attractiveness of all the cryptocurrencies. All three cryptocurrencies have positive weights in the optimal portfolio. Bitcoin has the largest weight, followed by Ripple and Litecoin. In the recent subsample, the contributions of the cryptocurrencies become more selective. Bitcoin weight increases, Ripple weight stays positive, while Litecoin weight is negative in the optimal portfolio in Panel B. We see that the contributions of the cryptocurrencies to an optimal portfolio evolve over time, indicating the highly dynamic nature of these investments.

For further verification of the dynamic evolution of optimal weights and cryptocurrency, we split our sample period into three sub-samples (early: August 2013-May 2015, middle: June 2015-March 2017, recent: April 2017-January 2019) and executed the optimization process to get the optimal weights, return, risk and $\mathrm{CV}$. The Bitcoin weights change as 0.9 , 5.9 and 6.5 percent for early, middle and recent subsamples (for Ripple the portfolio weight evolves as 0.6, -1.2 and 1.2 percent; and for Litecoin as 0.5, 0.3 and 0.4 percent). Moreover, using the optimal portfolio weights of the previous sub-sample for the next sub-sample generates a portfolio return of $3.3 \mathrm{bp}$ for the middle sub-sample (compared to the optimal $5.2 \mathrm{bp}$ ) and $5.2 \mathrm{bp}$ for the recent sub-sample (compared to the optimal $15.6 \mathrm{bp}$ ). All these out-of-sample analyses indicate the evolving and dynamic nature of the markets where the conclusions of this study provide a guideline into the future, but no guarantees.

For robustness, we explore two different avenues. In the optimal portfolio construction, it is natural for some securities to have negative weights, i.e., they would be short-sold.

\begin{tabular}{|c|c|c|c|c|c|c|}
\hline Return (hn) & Std (bp) & $\mathrm{CV}$ & & & & \\
\hline 3.98 & 25.81 & 6.486407 & & & & \\
\hline \multicolumn{7}{|c|}{ Early optimal portfolio weights (\%) } \\
\hline BTC & XRP & LTC & DJIA & VIX & Real estate & Bonds \\
\hline 1.2672 & 0.2848 & 0.5968 & 37.7350 & 4.2141 & 5.8285 & 50.0735 \\
\hline \multicolumn{7}{|c|}{ Panel B. Recent optimal portfolio (April 2016-January 2019) } \\
\hline Return (bp) & Std (bp) & $\mathrm{CV}$ & & & & \\
\hline \multirow{2}{*}{\multicolumn{7}{|c|}{ Recent optimal portfolio weights (\%) }} \\
\hline & & & & & & \\
\hline BTC & XRP & LTC & DJIA & VIX & Real Estate & Bonds \\
\hline 5.0531 & 2.2336 & -1.5149 & 73.2371 & 6.0069 & -4.9375 & 19.9218 \\
\hline
\end{tabular}

Notes: Optimal portfolio characteristics with different cryptocurrencies are provided in the table for the early subsample (from August 4, 2013 through April 26, 2016) and for the recent subsample (from April 27, 2016 through January 17, 2019). Panel A presents the optimal portfolio characteristics for the early subsample and Panel B presents optimal portfolio characteristics for the recent subsample. The return and standard deviation of the optimal portfolio are listed as basis points along with the coefficient of variation. Optimal portfolio weights are given as percentages. BTC is Bitcoin, XRP is Ripple, LTC is Litecoin, DJIA is Dow Jones Industrial Average Index, Real Estate is the Vanguard Real Estate Index and Bonds is the Vanguard Total Bond Market Index 
JCMS

3,2

110

The short positions for these inferior securities would enable the portfolio to place heavier weight on more attractive securities. While short selling is an organic part of free and efficient markets, it is prohibited by regulators in some markets due to ethical (benefiting from the decline of security prices may be frowned upon), psychological (leading to herding behaviors of excessive selling), or other reasons. In markets where short selling is not allowed, securities cannot have negative weights in portfolios. As the first robustness investigation, we explore optimal portfolios with this extra constraint of no short selling. The results are presented in Table VI, Panel A. Litecoin and Real Estate do not have any contribution to the portfolio and have zero weights, but Bitcoin and Ripple do have positive contributions to the optimal portfolio during the sample period. As expected, with the extra no-short restriction, the $\mathrm{CV}$ of the portfolio is worse than that of the portfolio reported in Table III.

Second, we examine the dynamic behavior of the optimal portfolio before and after the cryptocurrency crash. Throughout the beginning of 2018, from January to the mid of February, all cryptocurrencies lost significant value. This 2018 cryptocurrency crash, also known as the Bitcoin crash or the great crypto crash, was the sell-off of many cryptocurrencies during the month from January 6, 2018 to February 6, 2018, during when the Bitcoin value fell by about 65 percent. The capitalization of the cryptocurrency market lost more than $\$ 300 \mathrm{bn}$ in the first quarter of 2018.

Panel B of Table VI first reports the features of the optimal portfolio constructed during pre-crash period from August 2013 through December 2017. Consistent with Panel A of Table V, all the cryptocurrencies with their positive weights contribute to the formation of the optimal portfolio. The period after the Bitcoin crash period of 2018, March 2018 through January 2019, has been of poor performance for equity markets and real estate markets. This is reflected to the negative weights of equity and real estate in the post-crisis portfolio. The largest weight goes to bonds. As for cryptocurrencies, both Bitcoin and Ripple have large negative weights, while Litecoin has a positive weight in the portfolio - while the then popular Bitcoin and Ripple lose confidence, the demand for the upcoming Litecoin increases. The results in Panel B again indicate the dynamic and evolving characteristics of the

Panel A. No short-selling optimal portfolio

$\begin{array}{lcc}\text { Return (bp) } & \text { Std (bp) } & \text { CV } \\ 4.57 & 29.94 & 6.549303\end{array}$

No shot-selling optimal portfolio weights (\%)

$\begin{array}{llllll}\text { BTC XRP } & \text { LTC } & \text { DJIA } & \text { VIX } & \text { Real Estate } & \text { Bonds }\end{array}$

$\begin{array}{llllll}2.0911 & 0.8913 & 0.0000 & 43.6204 & 3.8765 & 0.0000\end{array}$

Panel B. Cryptocurrency crash: before and after

Before crash optimal portfolio weights (\%) (August 2013-December 2017)

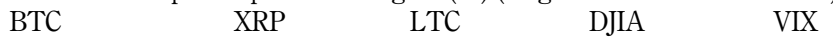

$\begin{array}{llll}2.3132 & 0.6262 & 0.2576 & 44.9937\end{array}$

After crash optimal portfolio weights (\%) (January 2018-January 2019)

BTC XRP LTC DJA VIX

$\begin{array}{lllll}-2.1030 & -10.7133 & 16.4655 & -109.8325 & -15.1745\end{array}$

$\begin{array}{cc}\text { Real Estate } & \text { Bonds } \\ -1.0181 & 49.2242 \\ & \\ \text { Real Estate } & \text { Bonds } \\ -3.6239 & 224.9817\end{array}$

Notes: Various robustness test results are reported. Panel A is about markets and time periods where short-selling may not be possible. The optimal portfolio characteristics with different cryptocurrencies are provided in this panel where short-selling is prohibited. Panel B is about the Bitcoin and cryptocurrency market crash in the beginning of 2018 from January to February. Before crash optimal portfolio weights for the subsample from August 2013 through December 2017 are followed by after crash optimal portfolio weights for the subsample from January 2018 through January 2019. Optimal portfolio weights are given as percentages. BTC is Bitcoin, XRP is Ripple, LTC is Litecoin, DJIA is Dow Jones Industrial Average Index, Real Estate is the Vanguard Real Estate Index and Bonds is the Vanguard Total Bond Market Index

Table VI.

Robustness: no short-selling and the Bitcoin crash 
cryptocurrency market. The crash has clearly led to reduction in the attractiveness Cryptocurrencies of cryptocurrencies as investment alternatives; however, the dynamic characteristics of cryptocurrencies warrant their future demand in investment portfolios.

\section{Conclusion}

In this study, we investigate popular cryptocurrencies as individual investment opportunities and as components of the construction of an optimal portfolio. Our sample period covers most of the 2010s. We examine three popular cryptocurrencies: Bitcoin, Ripple and Litecoin. As an independent single investment, Ripple has had the highest return, followed by Litecoin and Bitcoin during the sample period. However, volatility of Ripple is also the highest, followed by those of Litecoin and Bitcoin. Combining risk and return together, coefficient of variations reveal that the best cryptocurrency has been Ripple, followed by Bitcoin and then by Litecoin. All these cryptocurrencies have been better than the traditional asset classes in the 2010s for investment purposes.

We examine the role of cryptocurrencies in an optimal portfolio. We document that adding a cryptocurrency has consistently helped the optimal portfolio achieve a better return-and-risk combination. Bitcoin has been the best cryptocurrency in this regard, followed by Ripple and Litecoin. We report that the contribution of the cryptocurrencies to an optimal portfolio is dynamic and, therefore, evolves over time: when we split our entire sample into two subsamples, we find that all three cryptocurrencies have a positive role in the optimal portfolio in the early subsample. In the recent subsample, the role of the cryptocurrencies is not unidirectional. The Bitcoin crash has led to reduction in the attractiveness of cryptocurrencies as investment alternatives but their dynamic characteristics warrant their future demand for investment portfolios. We conclude in our study that popular cryptocurrencies have had an important role in portfolio construction and in investments, in addition to their original purposes for which they have been created.

As for the potential issues in the data analysis and for future avenues for research, we note that cryptocurrencies are still relatively new. One of the most popular cryptocurrencies, Ethereum, could not be included in our investigation because of the lack of statistically sufficient data. Our sample period covered the great cryptocurrency crash, illustrating the high volatility of these assets. As more data become available, as cryptocurrencies become more stable, and as familiarity with cryptocurrencies increases, the long-term effects of these assets in the formation of the optimal portfolio will become more informative.

\section{References}

Andrianto, Y. and Diputra, Y. (2017), "The effect of cryptocurrency on investment portfolio effectiveness", Journal of Finance and Accounting, Vol. 5 No. 6, pp. 229-238.

Anyfantaki, S., Arvanitis, S. and Topaloglou, N. (2018), "Diversification, integration and cryptocurrency market”, working paper, Bank of Greece, Athens, March 1.

Biais, B., Bisière, C., Bouvard, M. and Casamatta, C. (2019), "The blockchain folk theorem”, Review of Financial Studies, Vol. 32 No. 5, pp. 1662-1715.

Borri, N. (2019), "Conditional tail-risk in cryptocurrency markets", Journal of Empirical Finance, Vol. 50 No. C, pp. 1-19.

Bouri, E., Gupta, R. and Roubaud, D. (2019), "Herding behaviour in cryptocurrencies", Finance Research Letters, Vol. 29 No. C, pp. 216-221.

Brauneis, A. and Mestel, R. (2019), "Cryptocurrency-portfolios in a mean-variance framework", Finance Research Letters, Vol. 28 No. C, pp. 259-265.

Briere, M., Oosterlinck, K. and Szafarz, A. (2015), "Virtual currency, tangible return: portfolio diversification with Bitcoin”, Journal of Asset Management, Vol. 16 No. 6, pp. 365-373. 
JCMS

3,2

Burgess, R. (2018), "Ups, downs of investing in Bitcoin", available at: www.theindianalawyer.com/ articles/45940-the-ups-and-downs-of-investing-in-bitcoin (accessed December 15, 2018).

Cong, L.W. and He, Z. (2019), "Blockchain disruption and smart contracts", Review of Financial Studies, Vol. 32 No. 5, pp. 1754-1797.

Corbet, S., Meegan, A., Larkin, C., Lucey, B. and Yarovaya, L. (2018), "Exploring the dynamic relationships between cryptocurrencies and other financial assets", Economic Letters, Vol. 165 No. C, pp. 28-34.

Elendner, H., Trimborn, S., Ong, B. and Lee, T.M. (2018), "The cross-section of crypto-currencies as financial assets: investing in crypto-currencies beyond Bitcoin", in Chuen, D.L.K. and Deng, R. (Eds), Handbook of Blockchain, Digital Finance, and Inclusion, Academic Press, Cambridge, MA and Elsevier, Amsterdam, pp. 145-173.

Foley, S., Karlsen, J.R. and Putniňš, T.J. (2019), "Sex, drugs, and Bitcoin: how much illegal activity is financed through cryptocurrencies?”, Review of Financial Studies, Vol. 32 No. 5, pp. 1798-1853.

Geiregat, S. (2018), “Cryptocurrencies are (smart) contracts”, Computer Law \& Security Review, Vol. 34 No. 5, pp. 1144-1149.

Härdle, W.K. and Trimborn, S. (2015), "CRIX or evaluating blockchain based currencies", Working Paper No. 2015-048, SFB 649 Economic Risk, Berlin, October 15.

Klabbers, S. (2017), "Bitcoin as an investment asset", working paper, Radboud Universiteit Nijmegen, Nijmegen, June 2.

Klein, T., Thu, H.P. and Walther, T. (2018), "Bitcoin is not the new gold - a comparison of volatility, correlation, and portfolio performance", International Review of Financial Analysis, Vol. 59 No. C, pp. 105-116.

Kroll, Y., Levy, H. and Markowitz, H.M. (1984), "Mean-variance versus direct utility maximization", Journal of Finance, Vol. 39 No. 1, pp. 47-61.

Levy, H. and Markowitz, H.M. (1979), "Approximating expected utility by a function of mean and variance", American Economic Review, Vol. 69 No. 3, pp. 308-317.

Liu, W. (2019), "Portfolio diversification across cryptocurrencies", Finance Research Letters, Vol. 29 No. C, pp. 200-205.

Markowitz, H. (1952), "Portfolio selection”, Journal of Finance, Vol. 7 No. 1, pp. 77-91.

Merton, R.C. (1990), Continuous-Time Finance, Blackwell, Oxford.

Nakamoto, S. (2008), "Bitcoin - a peer-to-peer electronic cash system”, available at https://bitcoin.org/ bitcoin.pdf (accessed October 15, 2018).

Radovanov, B., Marcikić, A. and Gvozdenović, N. (2018), "A time series analysis of four major cryptocurrencies”, Facta Universitatis - Economics and Organization, Vol. 15 No. 3, pp. 271-278.

Velde, F. (2013), "Bitcoin: a primer”, Chicago Fed Letter No. 317, Chicago.

Wu, C.Y. and Pandey, V.K. (2014), "The value of Bitcoin in enhancing the efficiency of an investor's portfolio", Journal of Financial Planning, Vol. 27 No. 9, pp. 44-52.

\section{Corresponding author}

A. Can Inci can be contacted at: ainci@bryant.edu

For instructions on how to order reprints of this article, please visit our website:

www.emeraldgrouppublishing.com/licensing/reprints.htm

Or contact us for further details: permissions@emeraldinsight.com 